\title{
AVALIAÇÃO DA QUALIDADE DE VIDA EM PACIENTES IDOSOS UM ANO APÓS O TRATAMENTO CIRÚRGICO DE FRATURAS TRANSTROCANTERIANAS DO FÊMUR
}

\author{
FUNCTIONAL OUTCOMES AND QUALITY OF LIFE ONE YEAR AFTER SURGICAL \\ MANAGEMENT OF TROCHANTERIC FRACTURES
}

\begin{abstract}
Fernanda de Aquino Moraes Guimarães ', Renato Ribeiro de Lima², Alessandra de Castro Souza ${ }^{3}$, Bruno Livani ${ }^{4}$, William Dias Belangero ${ }^{5}$
\end{abstract}

\section{RESUMO}

Objetivo: Avaliar o impacto que as fraturas transtrocanterianas produzem sobre a qualidade de vida de pacientes idosos, tratados cirurgicamente, durante o período de um ano de acompanhamento. Material e Método: Foram selecionados 73 idosos com fratura transtrocanterina, com idade igual ou superior a 65 anos de ambos os sexos. Foi aplicado questionário padronizado, para se obter informações quanto aos hábitos de vida, atividade física, funcionalidade, deambulação e estado cognitivo. Foram excluídos os óbitos ocorridos durante o estudo, pacientes que não deambulavam, com doenças neurológicas ou fraturas patológicas. Resultados: A média de idade foi $80,17 \pm 7,2$, sendo $75 \%$ do sexo feminino. Ao comparar as somatórias das atividades de vida diária $(\mathrm{p}=0,04)$ e instrumentais da vida diária $(\mathrm{p}=0,004)$, obtidas na pré e pós-fratura, os pacientes tornaram-se mais dependentes pós-fratura. Atividades de vida diária que apresentaram piora pós-fratura foram: tomar banho $(p=0,04)$, ir ao banheiro $(p=0,02)$ e vestir-se $(p=0,04)$. Todas as atividades instrumentais da vida diária apresentaram diferença significativa, apresentando maior dependência funcional pós-fratura, assim como aumento da necessidade de auxílio a deambulação $(\mathrm{p}=0,00002)$, idade avançada $(\mathrm{p}=0,01)$ e não realizar atividades domésticas $(\mathrm{p}=0,01)$. A baixa pontuação no teste Minimental estava associada com uma maior dependência para realizar as atividades da vida diária na pré-fratura $(\mathrm{p}=0,00002)$ e pós-fratura $(\mathrm{p}=0,01)$. Conclusão: Após um ano, as atividades de vida diária que dependiam dos membros inferiores pioraram significativamente, todas as atividades instrumentais de vida diária apresentaram piora significativa em mais de $50 \%$ dos pacientes e mais da metade dos pacientes que andavam sem apoio perderam esta capacidade.

Descritores - Fratura Trocanteriana do Femur; Atividades Cotidianas; Idoso; Atividade Motora; Deambulação com Auxilio

\section{ABSTRACT}

Objective: Evaluate the impact that trochanteric hip fractures produce on life quality of elderly patients, treated surgically, during one year of monitoring. Methods: Were selected $73 \mathrm{el}-$ derly with transtrochanteric femur fracture, aged equal or over than 65 years and of both sexes. All participants underwent a standardized questionnaire which obtained information on lifestyle habits, functionality, physical activity, ambulation and cognitive state. Were excluded patients who died, not walking, with neurological diseases and pathological fractures. Results: The average age was $80.17 \pm 7.2$ years and $75 \%$ was female. When comparing the summation of activities of daily living $(p=0.04)$ and instrumental daily living $(p=0.004)$ obtained before and after fracture, the patients become more dependent after fracture. Activities of daily living that worsened after fracture were bathing $(p=0.04)$, toileting $(p=0.02)$ and dressing $(p=0.04)$. All instrumental activities of daily living showed a significant difference, with functional dependence after fracture, increased need for walking aid $(p=0.00002)$, aged $(p=0.01)$ and not doing housework $(p=0.01)$. The low score on the test Minimental was associated with a greater dependence to perform activities of daily living before ( $p=$ $0.00002)$ and after $(p=0.01)$ fracture. Conclusion: After a year, activities of daily living that depended on the lower limbs worsened significantly, all instrumental activities of daily living were significantly worse in more than $50 \%$ of patients and more than half of patients who walked without support lost this ability.

Keywords - Trocantheric Hip Fracture; Daily Activities; Ancient; Functional Activity; Dependent Ambulation

\footnotetext{
1 - Mestre em Ciências pela UNICAMP

2 - Doutor em Estatística e experimentação Agronômica ESALQ/USP e professor adjunto de Departamento de Ciência Exatas da UFLA.

3 - Doutora em Fisioterapia pela UFSCAR e professora do UNILAVRAS.

4 - Doutor pela FCM UNICAMP, médico do Hospital das Clínicas e Prof.Dr. PUC Campinas

5 - Livre Docente e Prof. Associado MS5 do Departamento de Ortopedia e Traumatologia da FCM/UNICAMP
}

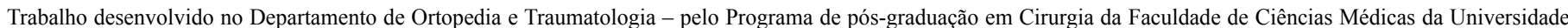

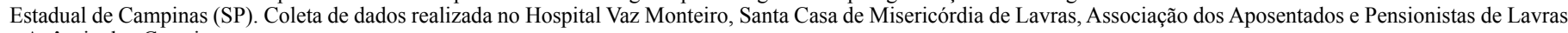
e Agência dos Correios.

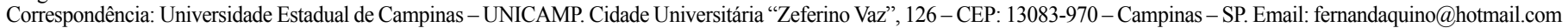

Trabalho recebido para publicação: 15/03/11, aceito para publicação: 15/04/11. 


\section{INTRODUÇÃO}

As fraturas do quadril e principalmente as fraturas da região transtrocanteriana do fêmur tiveram um aumento em sua incidência em todo o mundo devido ao envelhecimento da população ${ }^{(1,2)}$ Estas fraturas são consideradas hoje como um dos maiores problemas de saúde pública do século XXI atingindo a população idosa e do gênero feminino ${ }^{(3)}$.

A incidência destas fraturas aumenta após os 60 anos, com pico entre 70 e 79 anos, devido à osteoporose $\mathrm{e}^{(1,4)}$. A média de idade dos pacientes com fraturas transtrocanterianas (FTF) tende a ser 10 a 12 anos maior do que a dos pacientes com fratura do colo do fêmur ${ }^{(1,2)}$.

Sabe-se que a idade, o sexo, o uso de drogas psicotrópicas, abuso de álcool, tabaco, osteoporose, menopausa precoce, sedentarismo, incapacidade física, perda de equilíbrio, perda da capacidade cognitiva, presença de comorbidades são considerados fatores de risco para as quedas, fraturas e óbito ${ }^{(1,3,5)}$.

A maioria dos estudos foca, principalmente, a mortalidade que é elevada nesta população. No entanto, os estudos que avaliam a morbidade, ou seja, a perda de função pós-fratura são menos comuns ${ }^{(6)}$.

Por outro lado, os pacientes que sofrem FTF apresentam características próprias como menor massa óssea ${ }^{(2)}$ e maior limitação física medida pelas atividades da vida diária (AVD) e instrumentais da vida diária (AIVD), e capacidade de deambulação ${ }^{(4)}$.

O objetivo deste estudo foi avaliar o impacto que as fraturas transtrocanterianas produzem sobre a evolução clínica de pacientes idosos, tratados cirurgicamente, durante o período de um ano de acompanhamento.

\section{CASUÍSTICA E MÉTODO}

Tipo de estudo: Estudo prospectivo descritivo

\section{Casuística}

Este estudo foi conduzido na cidade de Lavras (MG), nos hospitais Vaz Monteiro e Santa Casa de Misericórdia de Lavras.

O recrutamento dos pacientes foi realizado no período de fevereiro de 2007 a dezembro de 2008. Durante este período foram identificados 89 casos de FTF. Destes foram entrevistados 73 pacientes que atenderam os critérios de inclusão do estudo e foram excluídos 21 pacientes que vieram a óbito dentro do período de um ano de acompanhamento da FTF.

\section{Aplicação do questionário}

Os participantes do estudo foram avaliados por um questionário padronizado baseado no estudo de $\mathrm{Braga}^{(7)}$, sendo aplicado por um único pesquisador e respondido pelo paciente avaliado. As questões do questionário podiam ser respondidas com ajuda do acompanhante, exceto o teste Minimental que deveria ser respondido exclusivamente pelo paciente avaliado.

As perguntas contidas no questionário avaliavam a capacidade de realização das atividades de vida diária (AVD) atividades instrumentais de vida diária (AIVD), necessidade ou não para realizar a deambulação, hábitos de atividade física regular, capacidade de realizar atividades domésticas e grau de escolaridade. Após a aplicação do questionário os pacientes foram submetidos ao Teste Minimental para avaliação da capacidade cognitiva.

A aplicação do questionário foi realizada preferencialmente antes da cirurgia, porém na sua impossibilidade devido à intercorrências, alguns pacientes foram entrevistados após a cirurgia. Todos os participantes aceitaram participar da pesquisa por livre e espontânea vontade e assinaram o Termo de Consentimento Livre e Esclarecido. Os mesmos foram selecionados de acordo com os critérios de inclusão e exclusão previamente definidos. Ao completar um ano da FTF, os pacientes foram contactados por telefone.

Este projeto foi aprovado pela Comissão de Ética Experimental da Faculdade de Ciências Médicas da Universidade Estadual de Campinas conforme parecer $n^{\circ}$ 109/2007.

\section{Critérios de inclusão}

Pacientes de ambos os sexos, com idade igual ou superior a 65 anos, deambuladores, com diagnóstico de FTF unilateral e tratados cirurgicamente na cidade de Lavras (MG).

\section{Critérios de exclusão}

Pacientes com idade inferior a 65 anos, sem condições de deambulação antes da fratura, com diagnóstico de Acidente Vascular Cerebral (AVC) prévio ou doenças neurológicas, neuropatias periféricas ou fratura patológica por tumor ou metástase.

\section{Variáveis estudadas}

Atividades de vida diária (AVD): São atividades básicas do indivíduo (tomar banho, alimentar-se, levantar-se, ir ao banheiro, vestir-se e tomar remédio) ${ }^{(7)}$. A mensuração das mesmas foi realizada pela determinação de valores, sendo o valor um para cada atividade que os 
pacientes necessitavam de ajuda para realizar e o valor dois para as atividades que não necessitavam de ajuda. Os pontos de cada AVD foram somados, sendo a pontuação mínima seis e máxima 12, ou seja, quanto maior a pontuação mais independente era o paciente. $O$ valor total, somatória dos pontos, foi abreviado como SAVD, e reduzido a duas categorias baseado no cálculo da mediana, sendo subdividido em $\leq 11 \mathrm{e}>11$. As AVD avaliadas com um ano da FTF foram denominadas AVDPO, sendo a somatória dos pontos abreviada como SAVDPO, possuindo os mesmos valores da mediana da SAVD.

Atividades instrumentais da vida diária (AIVD): São atividades que necessitam de maior habilidade do indivíduo para administrar o ambiente em que vive (ir a lugares distantes, fazer compras, preparar refeição, cuidar do banheiro e realizar serviço doméstico). ${ }^{(7)}$ Para avaliação das AIVD foi estabelecido valores para cada critério, sendo o valor um para incapacidade, dois para aqueles que necessitavam de ajuda e três para os que não necessitavam de ajuda, apresentando valor mínimo de cinco e máximo de 15 pontos. O valor total, somatória dos pontos, foi abreviado como SAIVD, e foram reduzidos a duas categorias $\leq 10$ e $>10$, baseado no cálculo da mediana, e para a avaliação da somatória das AIVD pós-fratura foi denominada de SAIVDPO, sendo os valores da mediana $\leq$ cinco ou $>$ cinco.

Teste Minimental: Para avaliação do estado cognitivo dos pacientes aplicou-se o teste Minimental. O teste fornece informações sobre diferentes parâmetros cognitivos, contendo questões agrupadas em sete categorias com o objetivo de avaliar funções cognitivas específicas. A pontuação do teste pode variar de no mínimo 0 pontos, o qual indica o maior grau de comprometimento cognitivo e ao total máximo de 30 pontos, o qual, por sua vez, corresponde a melhor capacidade cognitiva. ${ }^{(8)}$

Atividade física: Os pacientes foram divididos em dois grupos, os que realizavam e os que não realizavam atividade. As atividades desenvolvidas pelos pacientes foram caminhada, academia, ginástica, hidroginástica, andar de bicicleta e outras.

Atividades domésticas: Os pacientes foram avaliados quanto a realização ou não de atividades domésticas diárias na pré-fratura.

Grau de escolaridade: Pacientes foram divididos em dois grupos analfabetos e alfabetizados.

Auxílio a deambulação: Pacientes foram divididos na fase pré e pós-fratura em: deambuladores sem auxílio e com auxílio. Na fase pós-fratura foi acrescentado o não deambulador na classificação.

\section{ANÁLISE ESTATÍSTICA}

Os dados foram inseridos em planilhas no programa Excel e a análise estatística foi realizada no programa $\mathrm{R}^{(9)}$.

Para comparação das variáveis auxílio a deambulação, AVD (SAVD) e AIVD (SAIVD) antes e com um ano da FTF, foi aplicado teste não paramétrico, Teste de Wilcoxon. A comparação das variáveis categóricas como estado cognitivo, grau de escolaridade, idade, atividade física e atividade doméstica antes da fratura, e auxílio a deambulação, SAVD e SAIVD antes e com um da FTF foram utilizados testes paramétricos, o Teste Qui-Quadrado e o Teste Exato de Fisher, sendo considerado significativo valor de $\mathrm{p} \leq 0,05$.

\section{RESULTADOS}

A média de idade dos pacientes foi $80,17 \pm 7,2$ anos, sendo a maioria da população do estudo do gênero feminino 39 (75\%). Durante o período de um ano de acompanhamento $21(28,7 \%)$ pacientes vieram a óbito e 52 (71,2\%) sobreviveram.

A comparação da somatória dos valores atribuídos nas AVD e nas AIVD na fase pré-fratura e com um ano da fratura mostraram que houve piora significativa $(p=0,004)$ dessas atividades, refletindo maior dependência funcional após um ano da FTF.

Os pacientes foram classificados quanto a necessidade de auxílio para realizar cada AVD (Tabela 1). Dentro do período de um ano da FTF os pacientes tornaram-se mais dependentes para as AVD do tipo tomar banho, ir ao banheiro e vestirem-se sozinhos. As atividades que dependem mais dos membros superiores mantiveram-se.

Com relação a comparação entre AIVD antes e após a FTF, a perda do desempenho dos pacientes foi mais evidente, já que todas as atividades avaliadas pioraram significativamente após um ano da fratura em mais de $50 \%$ dos casos (Tabela 2).

Quanto a capacidade de deambulação ficou evidente que houve piora significativa após um ano da FTF. Na pré-fratura 43 pacientes $(82,7 \%)$ não necessitavam de auxílio à deambulação. Após um ano da FTF este número foi reduzido praticamente a metade já que apenas 23 pacientes $(44,2 \%)$ mantiveram sua capacidade de deambulação sem auxílio. Todavia, aumentou o número de pacientes que passaram a necessitar de auxílio a deambulação, 23 (44,2\%), enquanto seis $(11,5 \%)$ pacientes não recuperaram sua capacidade de deambular $(\mathrm{p}=0,00002)$ pós-fratura (Figura 1$)$. 
Tabela 1 - Distribuição dos pacientes quanto a necessidade ou não de auxílio para realizar as AVD na fase pré-fratura e após um ano de seguimento.

\begin{tabular}{c|c|c|c|c|c}
\hline \multirow{2}{*}{ AVD } & \multicolumn{2}{|c|}{ Pré-fratura } & \multicolumn{2}{c}{ Dentro de um ano da fratura } & P $\leq$ 0,05 \\
\cline { 2 - 5 } & Necessita & Não necessita & Necessita & Não necessita \\
\hline Tomar banho & $16(30,8 \%)$ & $36(69,2 \%)$ & $26(50 \%)$ & $26(50 \%)$ \\
\hline Alimentar-se & $2(3,8 \%)$ & $50(96,1 \%)$ & $3(5,8 \%)$ & $49(94,2 \%)$ & 0,04 \\
\hline Levantar-se & $6(11,5 \%)$ & $46(88,5 \%)$ & $12(23 \%)$ & $40(76,9 \%)$ & $37(71,1 \%)$ \\
\hline Ir banheiro & $6(11,5 \%)$ & $46(88,5 \%)$ & $15(28,8 \%)$ & 0,12 \\
\hline Vestir-se & $8(15,4 \%)$ & $44(84,6 \%)$ & $17(32,7 \%)$ & $35(67,3 \%)$ & 0,02 \\
\hline Medicar-se & $24(46,2 \%)$ & $28(53,8 \%)$ & $29(55,8 \%)$ & $23(44,2 \%)$ & 0,04 \\
\hline
\end{tabular}

Tabela 2 - Classificação dos pacientes quanto a necessidade de auxílio para realizar as AIVD, na pré-fratura e dentro do período de um ano de acompanhamento.

\begin{tabular}{c|c|c|c|c|c|c|c}
\hline \multirow{2}{*}{ AIVD } & \multicolumn{3}{|c|}{ Pré-fratura } & \multicolumn{3}{c}{ Dentro de um ano da fratura } & \multirow{2}{*}{ P $\leq \mathbf{0 , 0 5}$} \\
\cline { 2 - 8 } & Necessita & Não necessita & Incapaz & Necessita & Não necessita & Incapaz & \\
\hline Ir a lugares distantes & $16(30,8 \%)$ & $15(28,8 \%)$ & $21(40,4 \%)$ & $5(9,8 \%)$ & $8(15,7 \%)$ & $38(74,5 \%)$ & 0,003 \\
\hline Fazer compras & $7(13,5 \%)$ & $21(40,4 \%)$ & $24(46,2 \%)$ & $6(11,8 \%)$ & $10(19,6 \%)$ & $35(68,6 \%)$ & 0,02 \\
\hline Preparar refeições & $7(13,5 \%)$ & $32(61,5 \%)$ & $13(25 \%)$ & $3(5,9 \%)$ & $18(35,3 \%)$ & $30(58,8 \%)$ & 0,002 \\
\hline Serviço doméstico & $6(11,5 \%)$ & $24(46,2 \%)$ & $22(42,3 \%)$ & $5(9,8 \%)$ & $14(27,5 \%)$ & $32(62,7 \%)$ & 0,05 \\
\hline Cuidar do banheiro & $5(9,6 \%)$ & $24(46,2 \%)$ & $23(44,2 \%)$ & $6(11,8 \%)$ & $13(25,5 \%)$ & $32(62,7 \%)$ & 0,05 \\
\hline
\end{tabular}

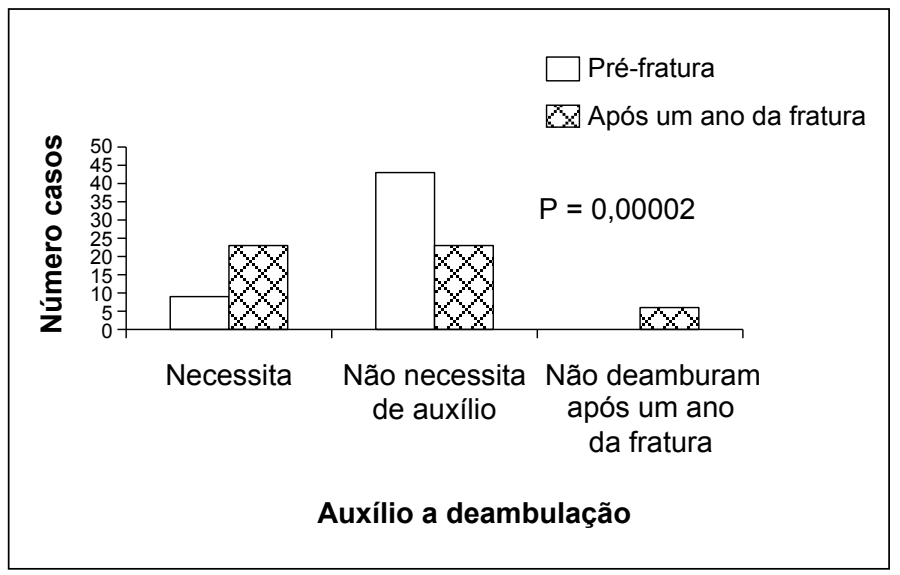

Figura 1 - Classificação dos pacientes quanto a necessidade de auxílio a deambulação, na fase pré-fratura e após um ano.

A influência da variável idade igual ou superior a 80 anos foi analisada como fator independente na recuperação pós-fratura das AVD e AIVD. A recuperação da AIVD não foi influenciada significativamente pela idade $(p=0,33)$, porém influenciou nas AVD ( $\mathrm{p}=0,01)$ (Tabela 3$)$.

Pelo Teste Exato de Fisher a necessidade de auxílio a deambulação foi avaliada em função da realização ou não de atividade física regular antes da FTF. Pela análise foi observado que a realização de atividade física antes da fratura não influenciou na recuperação da deambulação após um ano da FTF ( $\mathrm{p}=0,66)$.
Tabela 3 - Análise da influencia da idade na recuperação das AVD e das AIVD após um ano da FTF.

\begin{tabular}{c|c|c|c}
\hline \multirow{2}{*}{ Funcionalidade } & \multicolumn{2}{|c|}{ Idade } & \multirow{2}{*}{$\mathbf{P}<0,05$} \\
\cline { 2 - 3 } & $<\mathbf{8 0}$ anos & $\geq \mathbf{8 0}$ anos & \\
\hline SAVD $\leq 11$ & $13(25 \%)$ & $22(42,3 \%)$ & \multirow{2}{*}{0,01} \\
\hline SAVD $>11$ & $13(25 \%)$ & $4(7,7 \%)$ & \\
\hline SAIVD $\leq 5$ & $11(21,5 \%)$ & $16(31,4 \%)$ & \multirow{2}{*}{0,33} \\
\hline SAIVD $>5$ & $14(27,4 \%)$ & $10(19,6 \%)$ & \\
\hline
\end{tabular}

Legenda: SAVD (Somatória das atividades de vida diária); SAIVD (Somatória das atividades instrumentais da vida diária).

A recuperação da independência funcional (AVD e AIVD) durante o período de um ano da FTF foi avaliada em função da realização de atividades domésticas e atividade física regular, realizadas antes da fratura, pelo Teste de Homogeneidade Quiquadrado. Comparando os pacientes que realizavam atividade física regular com os que não realizavam não foi observada diferença significativa tanto para a recuperação pós-fratura das SAVD $(p=0,66)$ quanto para as SAIVD $(p=0,79)$. Quanto as atividades domésticas a maioria dos pacientes que não as realizavam tornaram-se mais dependentes na SAIVD $(p=0,01)$, porém o mesmo não foi observado com relação a SAVD $(p=0,26)$. 
A pontuação atingida no Teste Minimental, durante a primeira abordagem, foi comparada com objetivo de observar a influência do cognitivo na recuperação da independência para realizar as SAIVD e SAVD com um ano da FTF, como pode ser observado na tabela 4. Não foi observada diferença significativa para a SAIVDPO $(p=0,21)$, entretanto foram significativas as diferenças para a AVDPO $(\mathrm{p}=0,01)$.

Tabela 4 - Recuperação da independência funcional, AVD e AIVD, com ano da FTF em função da pontuação atingida no teste Minimental.

\begin{tabular}{c|c|c|c|c|c}
\hline \multirow{2}{*}{ Função } & \multirow{2}{*}{ Teste } & \multicolumn{3}{|c|}{ Pontuação Teste Minimental } & \multirow{2}{*}{$\mathbf{P}<\mathbf{0 , 0 5}$} \\
\cline { 3 - 5 } & & $\leq \mathbf{1 3}$ & $\mathbf{1 3}<\mathbf{x} \leq \mathbf{1 8}$ & $>\mathbf{1 8}$ & \\
\hline SAIVDPO $\leq 5$ & X2 & $9(17,6 \%)$ & $8(15,7 \%)$ & $10(19,6 \%)$ & \multirow{2}{*}{0,21} \\
\hline SAIVDPO > 5 & X2 & $3(5,9 \%)$ & $10(19,6 \%)$ & $11(21,6 \%)$ & \\
\hline SAVDPO $\leq 11$ & EF & $11(21,1 \%)$ & $14(26,9 \%)$ & $10(19,2 \%)$ & \multirow{2}{*}{0,01} \\
\hline SAVDPO > 11 & EF & $1(1,9 \%)$ & $4(7,7 \%)$ & $12(23 \%)$ & \\
\hline
\end{tabular}

Legenda: SAIVDPO (Somatória das atividades instrumentais da vida diária no pós-operatório); SAVDPO (Somatória das atividades de vida diária no pós-operatório)

O grau de escolaridade foi avaliado em função da pontuação atingida no teste Minimental, pois de acordo com a literatura quanto menor o grau de escolaridade menor será a pontuação atingida no teste Minimental. ${ }^{(54)}$ Neste estudo aproximadamente $82,7 \%$ dos pacientes eram alfabetizados. Após a análise estatística não foi observada diferença significativa entre o grau de escolaridade e a pontuação atingida no teste Mini-Mental $(p=0,07)$.

\section{DISCUSSÃO}

A literatura aponta que a recuperação da independência funcional após a fratura do quadril está associada com a ausência de demência, idade inferior a 85 anos, e capacidade para deambular e realizar as AVD e AIVD sem auxílio. ${ }^{(6,10-15)}$

No presente, ficou evidente que a FTF compromete a independência funcional e reduz significativamente as AVD e AIVD após um ano de seguimento. Somente $32,7 \%$ e $13,5 \%$ dos pacientes recuperaram as AVD e AIVD, respectivamente, após um ano, como eram na fase pré-fratura. Por outro lado, 5,7\% e 51,9\% dos pacientes tornaram-se dependentes para realizar as AVD e AIVD, respectivamente. Para Pillai et $a l^{(16)}$ entre os pacientes que eram independentes funcionalmente na fase pré-fratura $40,1 \%$ com idade entre 65 e 84 anos e $16,7 \%$ com idade $\geq 85$ anos recuperam a sua função após quatro meses da fratura indicando que a idade parece ter influencia nessa recuperação. No estudo de Fortes et $a l^{(17)}, 11,6 \%$ dos pacientes que sofreram fratura do quadril passaram a ser dependentes mesmo após seis meses da fratura e apenas 30\% recuperaram a capacidade de realizar as atividades que realizavam antes da fratura, sendo os fatores que mais influenciaram a perda da capacidade funcional foram a dependência funcional prévia à fratura, o número de comorbidades, a idade avançada, a perda da capacidade cognitiva e a baixa capacidade funcional no momento da alta hospitalar. No estudo de Garcia et al ${ }^{(18)}$ também ocorreu importante redução na capacidade funcional e maior dependência para realizar as AVD (tomar banho $55 \%$, alimentar-se $26 \%$, ir ao banheiro e vestir-se 48\%) e AIVD (fazer compras 94\%, preparar refeição $61 \%$, serviço doméstico $58 \%$, lavar roupa $55 \%$, tomar remédio $65 \%$ e usar transportes $81 \%$ ) após um ano da fratura do quadril. Achados semelhantes foram encontrados no estudo de Sepah et al ${ }^{(14)}$, que mostrou recuperação funcional das AVD em apenas $50 \%$ dos casos após seis meses.

Além dos fatores mencionados o gênero feminino, ausência de diabetes mellitus, capacidade de deambulação independente e não viver sozinho antes da fratura podem ser fatores de melhor prognóstico. ${ }^{(19)}$ Entretanto no estudo de Pillai et al ${ }^{(16)}$ apenas $7,7 \%$ e $1,8 \%$ dos pacientes que deambulavam antes da fratura com idade entre 65 a 84 anos e com idade igual ou superior a 85 anos, respectivamente, foram capazes de voltar a deambular sem auxílio após quatro meses da fratura. Segundo Sepah et $a l^{(14)} 76 \%$ dos pacientes que deambulavam na fase pré-fratura conseguiram recuperar a capacidade de deambulação após seis meses da cirurgia. No estudo de Pande et $a l^{(12)} 36 \%$ dos pacientes conseguiram deambular de forma independente após um ano da fratura do quadril, enquanto que $15 \%$ pararam de deambular. De acordo com Garcia et al ${ }^{(18)}$ dos pacientes que deambulavam antes da fratura, 36\% não conseguiram recuperar esta atividade mesmo após um ano da fratura do quadril.

No presente estudo apenas $23(44,2 \%)$ dos 43 $(88,5 \%)$ pacientes voltaram a deambular sem auxílio e seis pacientes $(11,5 \%)$ não conseguiram mais deambular mesmo com auxílio. Esta perda funcional tem efeito marcante na qualidade de vida desta população refletindo não só no comprometimento da capacidade motora 
como da propriocepção e do equilíbrio desses pacientes. Estes resultados indicam que há necessidade de se estudar com maior detalhamento o efeito da fratura na perda da qualidade da marcha para minimizar esta perda funcional no idoso.

A idade igual ou superior a 80 anos foi considerada como fator de pior prognóstico para a recuperação da capacidade da marcha e das AVD neste estudo. Na literatura esses fatores também são citados como fatores de piora da recuperação funcional ${ }^{(16,18)}$. No estudo de Kristensen et $\mathrm{l}^{(20)}$ a idade avançada estava independentemente associada a não recuperação da independência funcional no pós-operatório da fratura do quadril. Ao comparar pacientes com 80 e 70 anos a probabilidade de não recuparar a independência funcional foi de $50 \%$ e $60 \%$, respectivamente. Estes autores encontraram pela análise de regressão logística que a idade avançada, a presença de FTF e o baixo nível funcional na fase préfratura eram fatores associados significativamente com a perda de recuperação funcional ${ }^{(20)}$.

Os achados do presente estudo, são semelhantes aos da literatura ${ }^{(16,18,20)}$, em que a idade $\geq 80$ anos estava associada com uma maior dependência funcional para realizar as AVD e para deambular após a FTF.

A prática de atividade física regular é considerada como fator protetor da densidade mineral óssea, ${ }^{(3,11)}$ da resistência muscular, da qualidade da marcha e das respostas de proteção, diminuindo assim o risco de quedas $^{(3)}$. A independência funcional na fase pré-fratura também parece influenciar positivamente a recuperação funcional após a fratura do quadril no idoso ${ }^{(10)}$. De acordo com o estudo de Peel et a $l^{(11)}$ e Wei et $a l^{(3)}$ o risco de sofrer fraturas e as dificuldades de se recuperar após a fratura do quadril pode ser reduzido na população idosa com a realização de atividade física regular. Segundo Garcia et al ${ }^{(18)}$ somente $6,1 \%$ dos pacientes praticavam atividade física regular antes da fratura, e esta porcentagem diminuiu para 3,2\% após a fratura. A diminuição da atividade física nos idosos é considerada como causa e efeito da sua fragilidade, elevando o risco de fraturas do quadril, além de proporcionar maior dependência funcional e dificuldade para voltar a deambular na fase pós-fratura.

No presente estudo $63,5 \%$ dos pacientes não se exercitavam regularmente. Ao comparar os pacientes que realizavam atividade física, no período pré-fratura, com aqueles que não realizavam, não houve diferença significativa na recuperação funcional, medida pela das
SAVD $(\mathrm{p}=0,66)$ e SAIVD $(\mathrm{p}=0,79)$ após um ano. No entanto, os pacientes que realizavam atividades doméstica, antes da fratura, tornaram-se menos dependentes do que os que não realizavam, com relação aos parâmetros medidos na SAIVD $(\mathrm{p}=0,01)$.

De acordo com a literatura, indivíduos idosos com história de comprometimento do estado cognitivo ou com diagnóstico de demência, são mais susceptíveis a sofrer quedas e consequentemente fraturas do quadril ${ }^{(21)}$. Além disso, os idosos com comprometimento do estado cognitivo são mais dependentes funcionalmente, para realizar as AVD e AIVD ${ }^{(22,23)}$. Por outro lado o comprometimento do estado cognitivo pode também influenciar negativamente a recuperação funcional após a fratura do quadril. De acordo com o estudo de Samuelsson et $a l^{(22)} 95 \%$ dos pacientes que apresentavam cognitivo intacto eram independentes para realizar as AVD e os que apresentavam disfunção cognitiva (55\%) apresentavam dificuldade para deambular na fase pós-fratura. Em um estudo retrospecitivo, foi encontrado que dentre as comorbidades presentes, a demência, presente em $14 \%$ dos pacientes, foi a única variável que apresentou impacto negativo na sobrevida, na mobilidade e na independência para as AVD após seis meses da fratura do quadril $^{(15)}$. Com relação ao estudo de Gruber-Baldini et $a l^{(23)}, 50 \%$ dos pacientes apresentavam algum tipo de compromentimento do estado cognitivo na fase préfratura (28\%, ) eram mais dependentes nas AVD e AIVD. Essa disfunção foi mais frequente nos pacientes idosos, do sexo maculino, com baixo grau de escolaridade, com dependencia funcional (AVD e AIVD) quando comparado com pacientes sem alterações cognitivas. Segundo o estudo de Vidán et $a^{(13)}$, a recuperação funcional de pacientes idosos após a fratura do quadril foi melhor no grupo de pacientes que não apresentavam demência e naqueles que eram independentes para deambular na pré-fratura. Além disso, os pacientes que apresentaram capacidade cognitiva significativamente menor apresentaram comprometimento na recuperação funcional após um ano da fratura.

No presente estudo, os pacientes que obtiveram baixa pontuação no teste Minimental apresentaram maior dependência para as AVD, tanto no período pré-fratura $(p=0,00002)$ como na pós-fratura $(p=0,01)$, o que esta de acordo com a literatura.

Como pode ser observado nos resultados deste estudo, os fatores que influenciaram de forma negativa a evolução clínica dos pacientes foram o comprome- 
timento do estado cognitivo e a idade avançada. Ficou também evidente que pacientes com comprometimento da sua condição funcional medida pelas AVD e AIVD e capacidade de marcha foram os que mais sofreram influencia da fratura com piora dessa condição.

Sem dúvida a FTF repercute negativamente na condição de vida do idoso aumentando não só o risco de óbito, mas reduzindo praticamente pela metade a população apta a retomar sua vida nas mesmas condições que tinha na época pré-fratura. Sem dúvida tratamentos que utilizem técnicas que permitam reabilitação precoce devem ser a meta de todo cirurgião que lide com estes pacientes. Campanhas que conscientizem a população idosa da necessidade de realizar atividade física para melhorar sua independência funcional, equilíbrio, coordenação motora e propriocepção, também serão importantes fatores para melhorar a condição de marcha e reduzir o risco de queda e fratura. Realizar o diagnóstico da osteoporose e o seu tratamento no idoso com risco de sofrer fratura, bem como dos que sofreram deve ser obrigação de todo ortopedista que atenda esta população para evitar o primeiro ou novos episódio de fratura.

\section{CONCLUSÃO}

Após o período de um ano de acompanhamento de pacientes idosos com FTF, a evolução clinica, foi considerada pior nos mais idosos, com maior dependência funcional, que necessitavam de auxílio a deambulação, com comprometimento do estado cognitivo e com baixa capacidade física. Entretanto as atividades de vida diária que dependiam dos membros inferiores pioraram significativamente, todas as atividades instrumentais de vida diária apresentaram piora significativa em mais de $50 \%$ dos pacientes e mais da metade dos pacientes que andavam sem apoio perderam esta capacidade.

\section{REFERÊNCIAS}

1. Rocha MA, Carvalho WS, Zanqueta C, Lemos SC. Estudo epidemiológico retrospectivo das fraturas do fêmur proximal tratados no hospital escola da faculdade de medicina do Triângulo Mineiro. Rev Bras Ortop. 2001;36(8):311-15.

2. Gameiro VS, Schott PCM. A osteoporose em fraturas proximais de fêmur: estudo histomorfométrico. Rev Bras Ortop. 2006;41(3):61-9.

3. Wei TS, Hu CH, Wang SH, Hwang KL. Falll characterictics, funcional mobility and bone mineral density as risk factors of hip fracture in the community-dwelling ambulatory elderly. Osteoporos Int. 2001:12:1050-1055.

4. Koval KJ, Aharonoff GB, Rokito AS, Lyon T, Zuckerman JD. Patients with femoral neck and intertrochanteric fractures. Are they the same? Clin Orthop Relat Res. 1996;330:166-172.

5. Pereira SRM, Buksman S, Perracini M, Py L, Barreto KML, Leite VMM. Quedas em idosos. SBGG. 2001:1-9.

6. Johnell O, Kanis JA. An estimate of the worldwide prevalence and disability associated with osteoporotic fractures. Osteoporos Int. 2006;17(12):1726-1733.

7. Braga TBT. Uso de diuréticos, psicotrópicos e fraturas de quadril em idosos: um estudo caso-controle [Tese]. Campinas: Universidade Estadual de Campinas; 2002.

8. Chaves MLF. Testes de avaliação cognitiva: Mini-Exame do Estado Mental. Neurologia cognitiva e do envelhecimento da ABN. [periódico na internet]. 2006-2008. Disponível em: http://www.cadastro.abneuro.org/site/arquivos_cont/8.pdf.

9. R Development Core Team. R: A language and environment for statistical computing. R Foundation for Statistical Computing, Vienna, Austria. 2010. ISBN 3-900051-07-0. URL http://www.R-project.org.

10. Taylor BC, Schreiner PJ, Stone KL, Fink HA, Cummings SR, Nevitt MC, et al. Long-term prediction of incident hip fracture risk in elderly white women: study of osteoporotic fractures. J Am Geriatr Soc. 2004; 52(9):1479-1486.

11. Peel NM, McClure RJ, Hendrikz JK. Health-protective behaviours and risk of fall-related hip fractures: a population-based case-control study. Age Ageing. 2006;35(5):491-497.

12. Pande I, Scott DL, O'Neill TW, Pritchard C, Woolf AD, Davis MJ. Quality of life, morbidity and mortality after low trauma hip fracture in men. Ann Rheum Dis. 2006; 65: 87-92.
13. Vidán M, Serra JA, Moreno C, Riquelme G, Ortiz J. Efficacy of a comprehensive geriatric intervention in older patients hospitalized for hip fracture: A randomized, controlled trial. J Am Geriatr Soc. 2005;53:1476-1482.

14. Sepah YJ, Umer M, Khan A, Niazi AUK. Functional outcome, mortality and inhospital complications of operative treatment in elderly patients with hip fractures in the developing world. Int Orthop. 2010; 34(3): 431-435.

15. Penrod JD, Litke A, Hawkes WG, Mgaziner J, Doucette JT, Koval KJ, et al. The association of race, gender, and comorbidity with mortality and function after hip fracture. J Gerontol. 2008;63(8):867-872.

16. Pillai A, Eranki V, Shenoy R, Hadidi M. Age Related Incidence and Early Outcomes of Hip Fractures: A Prospective Cohort Study of 1177 patients. J Orthop Surg Res. 2011 Jan 24;6(1):5.

17. Fortes EM, Raffaelli MP, Bracco OL, Takata ETT, Reis FB, Santili C, et al. Elevada morbimortalidade e reduzida taxa de diagnóstico de osteoporose em idosos com fratura de fêmur proximal na cidade de São Paulo. Arq Bras Endocrinol Metab. 2008; 52(7):1106-1114.

18. Garcia R, Leme MD, Garcez-Leme LE. Evolution of brazilian elderly with hip fracture secondary to a fall. Clinics. 2006;61(6):539-44.

19. Semel J, Gray JM, Ahn HJ, Nasr H, Chen JJ. Predictors of outcome following hip fracture rehabilitation. PM\&R. 2010 Sep;2(9):799-805.

20. Kristensen MT, Foss NB, Ekdahl C, Kehlet $\mathrm{H}$. Prefracture functional level evaluated by the New Mobility Score predicts in-hospital outcome after hip fracture surgery. Acta Orthop. 2010; 81 (3): 296-302.

21. Formiga F, Navarro M, Duaso E, Chivite D, Ruiz D, Perez-Castejon JM, et al. Factors associated with hip fracture-related falls among patients with a history of recurrent falling. Bone. 2008; 43: 941-944.

22. Samuelsson $B$, Hedström MI, Ponzer $S$, Söderqvist $A$, Samnegård $E$, Thorngren $K G$, et al. Gender differences and cognitive aspects on functional outcome after hip fracture - A 2 years' follow-up of 2,134 patients. Age Ageing. 2009 Nov; 38(6):686-92.

23. Gruber-Baldini AL, Zimmerman S, Morrison S, Grattan LM, Hebel R, Dolan MM, et al. Cognitive Impairment in Hip Fracture Patients: Timing of Detection and Longitudinal Follow-Up. JAGS. 2003; 51:1227-1236. 